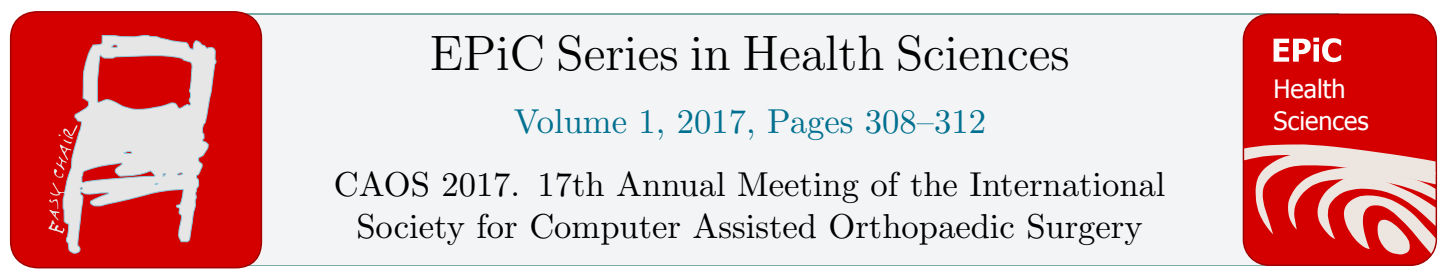

\title{
An Optimized Least-Moving-Point Algorithm to Detect the Hip Center
}

\author{
Guillaume Dardenne ${ }^{1,2}$, Zoheir Dib ${ }^{1,3}$, Chafiaa Hamitouche ${ }^{1,4}$, Christian \\ Lefèvre ${ }^{1,2,3}$ and Eric Stindel ${ }^{1,2,3}$ \\ ${ }^{1}$ LaTIM, INSERM, UMR 1101, SFR IBSAM, Brest, 29200, France. \\ ${ }^{2}$ CHU Brest, Brest, 29200, France. \\ ${ }^{3}$ Université de Bretagne Occidentale, Brest, 29200, France. \\ ${ }^{4}$ IMT-Atlantique, Dpt ITI, Brest, 29200, France. \\ guillaume.dardennelchu-brest.fr
}

\begin{abstract}
Functional approaches for the localization of the hip center (HC) are widely used in Computer Assisted Orthopedic Surgery (CAOS). These methods aim to compute the $\mathrm{HC}$ defined as the center of rotation (CoR) of the femur with respect to the pelvis. The Least-Moving-Point (LMP) method is one approach which consists in detecting the point that moves the least during the circumduction motion. The goal of this paper is to highlight the limits of the native LMP (nLMP) and to propose a modified version (mLMP). A software application has been developed allowing the simulation of a circumduction motion of a hip in order to generate the required data for the computation of the HC. Two tests have been defined in order to assess and compare both LMP methods with respect to (1) the camera noise (CN) and (2) the acetabular noise (AN). The mLMP and nLMP error is respectively: (1) $0.5 \pm 0.2 \mathrm{~mm}$ and $9.3 \pm 1.4 \mathrm{~mm}$ for a low $\mathrm{CN}, 21.7 \pm 3.6 \mathrm{~mm}$ and $184.7 \pm 13.1 \mathrm{~mm}$ for a high $\mathrm{CN}$, and (2) $2.2 \pm 1.2 \mathrm{~mm}$ and $0.5 \pm 0.3 \mathrm{~mm}$ for a low AN, $35.2 \pm 18.5 \mathrm{~mm}$ and $13.0 \pm 8.2 \mathrm{~mm}$ for a high AN. In conclusion, mLMP is more robust and accurate than the nLMP algorithm.
\end{abstract}

\section{Introduction}

Different functional approaches have been described in the literature in order to localize the Hip Center (HC) in computer assisted orthopedic surgery (Ehrig, 2006). The two main methods are the Pivot (PIV) (Siston, 2006) and the Least Moving Point (LMP) algorithms (Marin, 2003), (Stindel, 2005). Unlike PIV, the LMP method is based on the hypothesis that the HC must be considered as a mobile point during the rotation motion. Several parameters, such as the non-sphericity of the femoral head, the motion of the pelvis during the acquisition, or the error associated to the localization system, 
could impact the instantaneous position of the center of rotation during the motion. The functional $\mathrm{HC}$ is thus considered to be the point that moves the least. The goal of this paper is to highlight the limits of the native LMP (nLMP) method and to propose a modified LMP (mLMP) approach. The accuracy and precision of both methods have been assessed and compared in a simulated environment.

\section{Materials and Methods}

The HC obtained with the nLMP and called $H C_{n L M P}$ can be determined according to the following equation:

$$
f(c)=\sum_{i=2}^{N}\left\|c_{i-1}-c_{i}\right\|^{2}
$$

The mLMP approach adds two additional processing steps to the nLMP:

1. The main list containing the $l$ transforms $\left[T_{1}, T_{2}, \ldots, T_{l}\right]$ acquired during the circumduction motion with the navigation system is first sampled. From this main list, the sampling process creates thus $m$ sub-lists $\left[L_{1}, L_{2}, \ldots, L_{m}\right]$ which contain $i$ sampled transforms, with $i \ll l$. This sampling process is performed according to a distance $d_{\text {sampling }}$ so that the distance between two successive transforms $T_{j}$ and $T_{j+1}$ of a given sub-list is superior to $d_{\text {sampling. }}$. For each sub-list $L_{j}$, with $0<j<m, H C_{n L M P_{-} j}$ is computed by using the nLMP method. We thus obtain $m$ HCs associated to the $m$ sub-lists.

2. The hip center $H C_{m L M P}$ from the mLMP approach is then computed. An average HC, called $H C_{\text {init }}$ is first calculated from the $m$ previous HCs. A threshold operation is then realized, which aims to reject $k \mathrm{HCs}$, with $k<m$, from the $H C_{n L M P}$, having a distance superior to $d_{\text {threshold }}$ from $H C_{\text {init }}$. The final $H C_{m L M P}$ is therefore the average of the preserved $(m-k)$ HCs.

The entire process requires thus the initialization of two parameters: (a) the distance $d_{\text {sampling }}$ for the computation of the $m$ sub-lists, and (b) the distance $d_{\text {threshold }}$ allowing the rejection of the $k$ HCs. These parameters have been found experimentally and has been set respectively to $40 \mathrm{~mm}$ and $2 \mathrm{~mm}$ in order to optimize the $\mathrm{HC}$ detection.

The $l$ transforms have been generated thanks to a $\mathrm{C}++$ software application which can simulate the position and the orientation of a femoral tracker during a circumduction motion and with respect to a

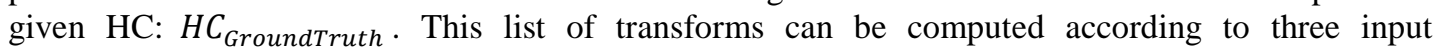
parameters (1) the number of laps (NL), (2) the camera noise (CN) representing the noise associated to the localization system and simulated by a Gaussian noise applied to the three dimensional (3D) positions and rotations of the transforms, and (3) the acetabular noise (AN) allowing the simulation of the non-sphericity of the femoral head or the motion of the pelvis during the acquisition and simulated also by a Gaussian noise applied to the three dimensional (3D) positions of the $H C_{\text {GroundTruth }}$.

Two tests have been defined in order to study the influence of the camera and the acetabular noises onto both nLMP and mLMP approaches:

- Test 1 - Camera noise: 
$\mathrm{CN}$ has been modified from $0.1^{\circ}$ to $1^{\circ}$ with a step of $0.1^{\circ}$ for the rotation, and from 0.5 $\mathrm{mm}$ to $5 \mathrm{~mm}$ with a step of $0.5 \mathrm{~mm}$ for the translation. In this configuration, NL, and AN have been respectively set to 1 and $0 \mathrm{~mm}$.

- Test 2 - Acetabular noise:

AN has been modified from $1 \mathrm{~mm}$ to $10 \mathrm{~mm}$ with a step of $1 \mathrm{~mm}$. NL has been set to 1 and 5. In this configuration, $\mathrm{CN}$ has been set to $0^{\circ}$ in rotation $/ 0 \mathrm{~mm}$ in translation.

The errors are defined as the distance between $H C_{\text {GroundTruth }}$ and $H C_{n L M P}$ for $\mathrm{nLMP}$, and between $H C_{\text {GroundTruth }}$ and $H C_{m L M P}$ for mLMP. All these tests have been performed 1,000 times. 150 transforms have been acquired per laps.

\section{Results}

Figure 1 shows the error of the test 1 . The error (mean $\pm \mathrm{SD}$ ) ranged from $0.5 \pm 0.2 \mathrm{~mm}$ and $9.3 \pm 1.4 \mathrm{~mm}$ with a $\mathrm{CN}$ of $0.5 \mathrm{~mm}$ in translation and $0.1^{\circ}$ in rotation, to $21.7 \pm 3.6 \mathrm{~mm}$ and $184.7 \pm 13.1$ $\mathrm{mm}$ with a $\mathrm{CN}$ of $5 \mathrm{~mm}$ in translation and $1^{\circ}$ in rotation, for respectively the mLMP and nLMP methods.

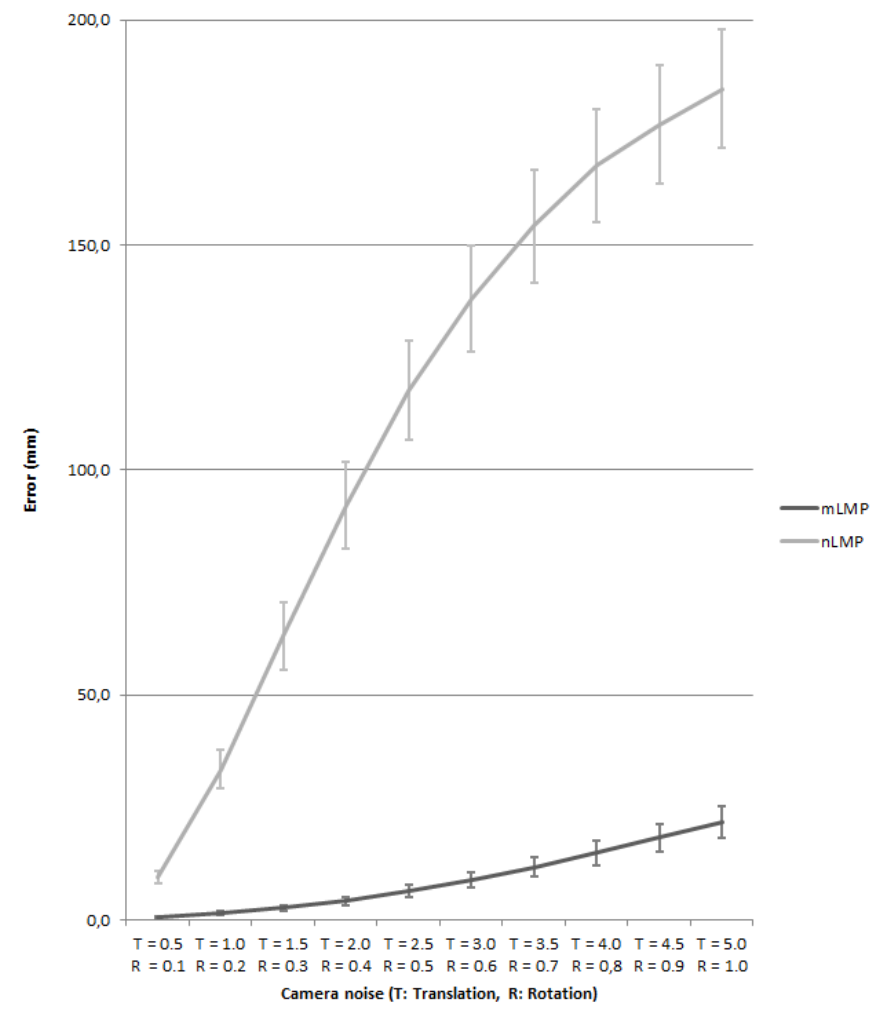

Figure 1: Error (mean $\pm \mathrm{SD}$ ) of the mLMP and nLMP methods according to the $\mathrm{CN}$ 
Figure 2 (a) shows the error of the test 2 for one lap. The error (mean \pm SD) ranged from $0.5 \pm 0.3$ $\mathrm{mm}$ and $2.2 \pm 1.2 \mathrm{~mm}$ with an AN of $1 \mathrm{~mm}$, to $13.0 \pm 8.2 \mathrm{~mm}$ and $35.2 \pm 18.5 \mathrm{~mm}$ with an AN of $10 \mathrm{~mm}$, for respectively the mLMP and nLMP methods.

Figure 2 (b) shows the error of the test 2 for five laps. The error (mean \pm SD) ranged from $0.2 \pm 0.1$ $\mathrm{mm}$ and $0.9 \pm 0.5 \mathrm{~mm}$ with an AN of $1 \mathrm{~mm}$, to $3.5 \pm 2.0 \mathrm{~mm}$ and $15.3 \pm 8.2 \mathrm{~mm}$ with an AN of $10 \mathrm{~mm}$, for respectively the mLMP and nLMP methods.

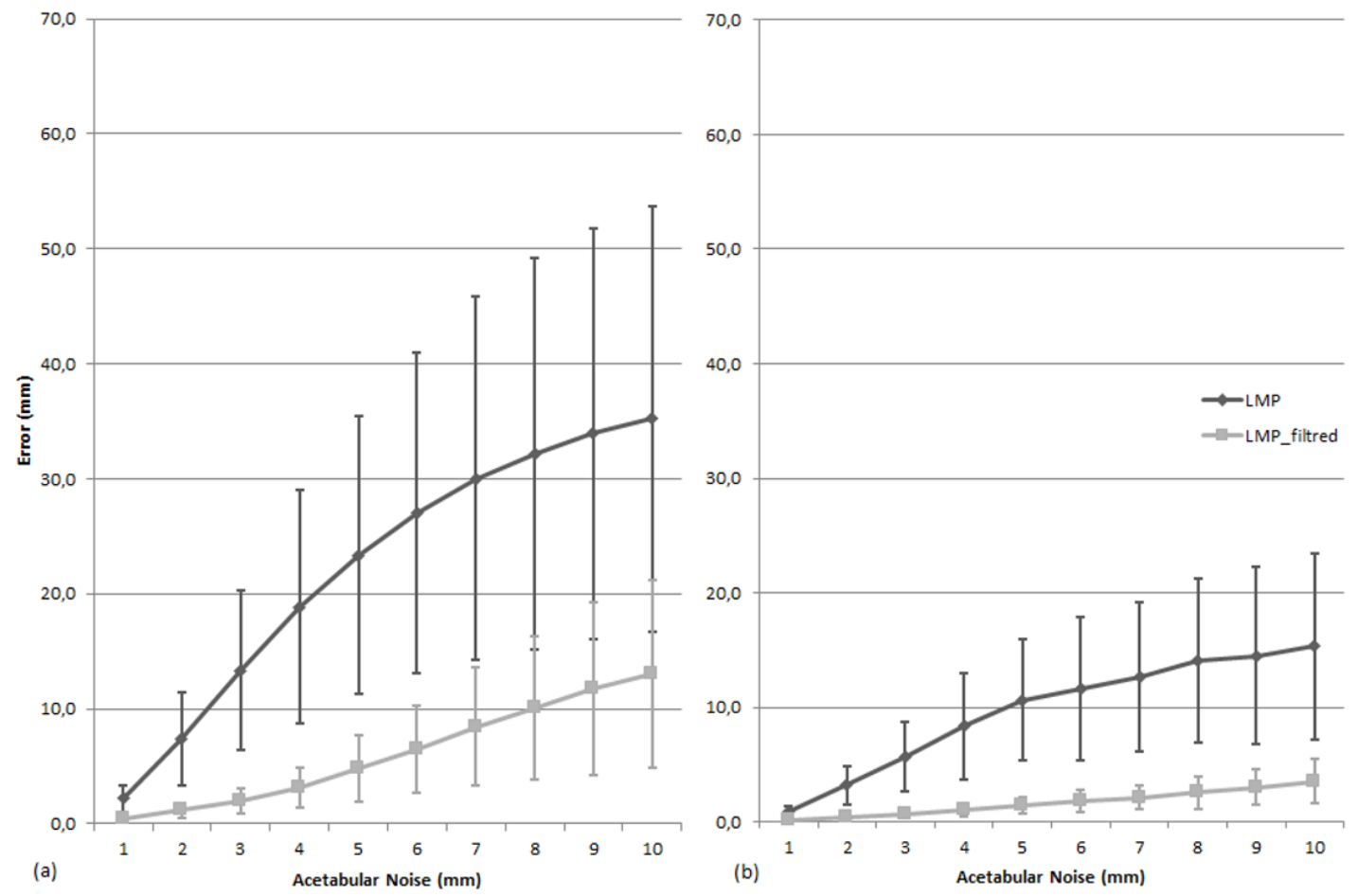

Figure 2: Error (mean \pm SD) of the mLMP and nLMP methods according to the AN for one lap (a) and five laps (b)

\section{Discussion}

Several factors can impact the localization of the functional HC (Stindel, 2005) (Ehrig, 2006). Among them, CN and AN are reported to be the most influent. The results from tests 1 and 2 confirm the sensitivity of both LMP approaches to these factors. However, we observed that the mLMP introduces an improvement in the accuracy of the detection, whatever the noise value. For extreme values of the camera noise, the improvement can be even 8 times superior for mLMP. The number of laps can also improve the accuracy. However, the mLMP approach is always more accurate than the nLMP. 


\section{References}

Dib, Z. (2013). Detection of the hip center in computer-assisted surgery: An in vitro assessement study. IRBM, 34, 319-321.

Ehrig, R. (2006). A survey of formal methods for determining the centre of rotation of ball joints. Journal of Biomechanics, 39(15), 2798-2809.

Marin, F. (2003). Accurate determination of a joint centre center based on the minimal amplitude point method. Computer Aided Surgery, 8, 30-34.

Siston, R. (2006). Evaluation of a new algorithm to determine the hip joint center. Journal of Biomechanics, 39(1), 125-30.

Stindel, E. (2005). Detection of the center of the hip joint in computer-assisted surgery: an evaluation study of the Surgetics algorithm. Computer Aided Surgery, 10(3), 133-9. 